\title{
A three-dimensional model of water circulation and temperature structure in the Caspian Sea
}

\author{
S. Nasimi ${ }^{1}$ \& R. Ghiassi ${ }^{2}$ \\ ${ }^{1}$ Islamic Azad University Gorgan, Iran \\ ${ }^{2}$ College of Civil Engineering Tehran, Iran
}

\begin{abstract}
This research is based on a numerical model for forecasting the threedimensional behaviour of (sea) water motion due to the effect of the variable wind velocity. The results obtained are then analyzed and compared with observations.

This model is based on the equations that overcome the current and distribution of temperature by applying the method of finite difference by assuming $\Delta \mathrm{x}, \Delta \mathrm{y}$ as constant and $\Delta \mathrm{z}$ as variable.

The model has been evaluated according to the reports and observations. Through studying the position of the current in different layers, the velocity in the cross section in the northern, southern and the middle layers will be discussed. The results reveal the presence of the circulation cells in the three above-mentioned areas. The circulation is reduced with increase in depth. The current velocity throughout the whole fields is different. The first layer current velocity is calculated to be between $1.6 \mathrm{~cm} / \mathrm{s}$ and $15 \mathrm{~cm} / \mathrm{s}$. It seems that the maximum velocity in the middle is considered by the maximum wind velocity $7 \mathrm{~m} / \mathrm{s}$ and wind stress $\left(\tau_{x}=\tau_{y}=0.07 \mathrm{~N} / \mathrm{m}^{2}\right)$. The results obtained through the numerical solution of the temperature equation have been compared with the observations. The temperature change in different layers in the cross section illustrates the relative harmony of the model mentioned.
\end{abstract}

Keywords: circulation, temperature, numerical model, variable wind stress. 


\section{Introduction}

The hydrodynamic processes influence the ecology and biology of the system directly. One of the most significant methods to estimate the hydrodynamic parameters is modeling.

Casandy (1971-73) and Bennet and Simins (1979), present an understanding of the limitations and stability of these models in large scales. The presentation was done through the analytical studies upon which the later researches on the lake modeling were conducted $[1,2,4,6,8,10]$. These models were mostly utilized for Michigan, Ontario, Erie, lakes. Hatter and Wang are among the researchers who studied the closed lakes and investigated about the threedimensional model of water circulation, resulting from the changing wind around Mainan Island [11]. Fadawi Hossaini's study is among the fields of wind stress in the Caspian Sea, it's stratification of permanent density, and coefficiency of coriolis parameter has been assumed as fixed [5].

The model forecasting and its quality in the Caspian Sea is deeply linked to the forecasting a complete atmospheric model, especially to wind, the in influence of friction and time. The present study will demonstrate a model being capable of showing the circulation and structure of temperature, as well as the variable wind patterns. This model, considering the vast horizontal grid including $10 \times 10 \mathrm{Km}$ extension and a vertical grid with a desirable separation in the upper layer, is able to demonstrate the details better in comparison with Hossaini's model [5].

\section{Boundary conditions and the equations}

The boundary conditions model presuppositions are as follows:

A. At the boundaries the velocity component is zero $(\mathrm{u} . \mathrm{n}=0, \mathrm{w}=0)$, except for the rivers flow.

B. The hydrostatic approximation is set up and the vertical accelerations have been ignored.

C. Approximation of $f$ plate is utilized by considering the length of the Caspian Sea, the variability of coriolis parameter, and the Cartesian coordinates.

D. The friction is determined through the viscosity of vertical and horizontal circulation.

E. At the base, the bottom stress has been measured by a linear drag coefficient formula.

F. Temperature distribution has been measured through the turbulence distribution.

G. Boussinesqu approximation: density variations are neglected except where they influence buoyancy.

By considering the above-mentioned factors, the equations are as follows:

$$
\frac{d U}{d t}=f V-\frac{1}{\rho_{\mathrm{o}}} \frac{\partial P_{s}}{\partial x}-\frac{1}{\rho_{\mathrm{o}}} \frac{\partial P}{\partial x}+\left[A_{h}\left(\frac{\partial^{2} U}{\partial x^{2}}+\frac{\partial^{2} U}{\partial y^{2}}\right)+A_{z} \frac{\partial^{2} U}{\partial z^{2}}\right]
$$




$$
\begin{gathered}
\frac{d V}{d t}=-f U-\frac{1}{\rho_{\circ}} \frac{\partial P_{s}}{\partial y}-\frac{1}{\rho_{\circ}} \frac{\partial P}{\partial y}+\left[A_{h}\left(\frac{\partial^{2} V}{\partial x^{2}}+\frac{\partial^{2} V}{\partial y^{2}}\right)+A_{z} \frac{\partial^{2} V}{\partial z^{2}}\right] \\
\frac{\partial(L \psi)}{\partial t}=-\left[U \frac{\partial}{\partial x}(L \psi)+V \frac{\partial}{\partial y}(L \psi)\right]-\beta \frac{\partial \psi}{\partial x}+A_{h} \nabla^{4} \psi+\frac{1}{\rho H}\left[\frac{\partial \tau_{s y}}{\partial x}+\frac{\partial \tau_{s x}}{\partial y}\right] \\
-\frac{1}{\rho H}\left[\frac{\partial \tau_{m y}}{\partial x}+\frac{\partial \tau_{m x}}{\partial y}\right]+\frac{1}{\rho H^{2}}\left(\tau_{z u p x}-\tau_{z d o w n x}\right) \frac{\partial H}{\partial y}-\frac{1}{\rho H^{2}}\left(\tau_{z u p y}-\tau_{z d o w n y}\right) \frac{\partial H}{\partial x} \\
\frac{\partial U}{\partial x}+\frac{\partial V}{\partial y}+\frac{\partial W}{\partial z}=0 \\
\frac{\partial T}{\partial t}=-\frac{\partial(U T)}{\partial x}-\frac{\partial(V T)}{\partial y}-\frac{\partial}{\partial z}\left(W T-K \frac{\partial T}{\partial z}\right)
\end{gathered}
$$

Equations (1) and (2) belong to the motion size for two components related to the horizontal velocity. Equation (3), curl equation and operator $L \psi$ is defined as:

$$
L \psi=\left[\frac{\partial}{\partial x}\left(\frac{\partial \psi}{\partial x}\right)+\frac{\partial}{\partial y}\left(\frac{\partial \psi}{\partial y}\right)\right]
$$

Equation (4) is the continuity equation, and equation (5) belongs to thermodynamic energy. All the location derivatives are approximated through the central difference. The horizontal velocity components, when repeated, can be measured by the following definition:

$$
\begin{aligned}
U^{n+1} & =-\frac{1}{n} \frac{\partial \psi^{n+1}}{\partial y} \\
V^{n+1} & =-\frac{1}{n} \frac{\partial \psi^{n+1}}{\partial x}
\end{aligned}
$$

The vertical velocity, when repeated, is measured by the following equation:

$$
\frac{\partial W^{n+1}}{\partial z}=-\frac{\partial U^{n+1}}{\partial x}-\frac{\partial V^{n+1}}{\partial y}
$$

\section{The Caspian Sea modeling resulting from the variable wind}

The experiments, considering the different boundary conditions and different wind fields, show that the computer programming is accurate. Therefore, it can be practically used for the Caspian Sea studies.

The computer programming is able to forecast the water circulation in a threedimensional form caused by the wind having a variable field. The surface horizontal grid and the middle horizontal layers cover a unit of $10 \times 10 \mathrm{Km}$. The vertical distance in the variable horizontal layers has been considered. The layers thicknesses, according to the metric system are 5, 10, 20, 30, 60, 100, 150, 250, 500 and more, from the upper parts to the bottom. In addition to the Variability 
of wind stress field, the density stability and the coefficient of distribution has been assumed as fixed.

The graphs 1, 2, 3 represent the vertical boundary, and grid according to numerical method of limited difference for the three-dimensional model in the Caspian Sea, both lengthwise and widthwise.
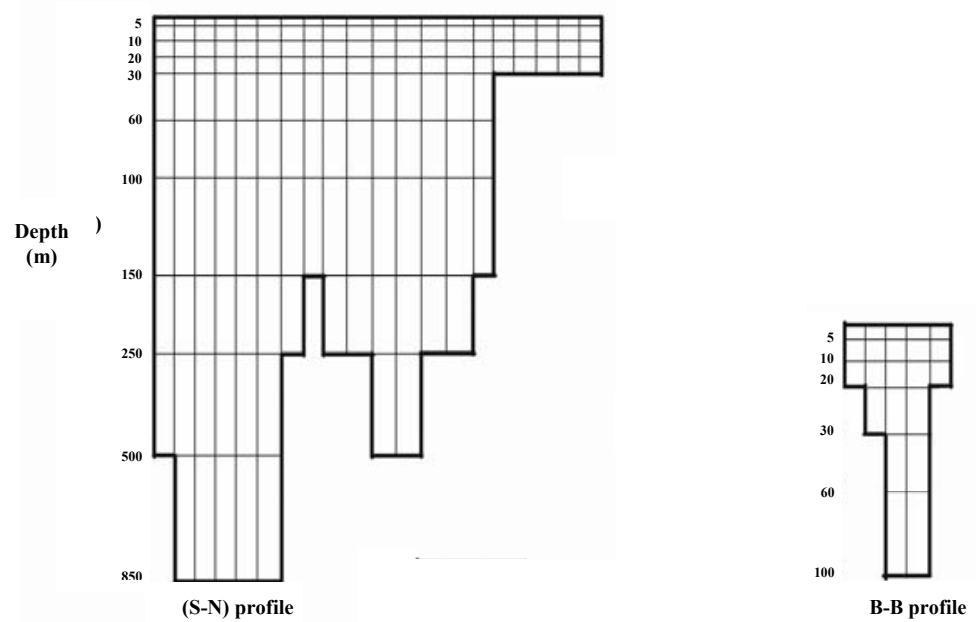

Figure 1: Vertical reticulating of Figure 2: Vertical reticulating limited difference (lengthwise direction). of limited difference (widthwise direction of Caspian Sea).

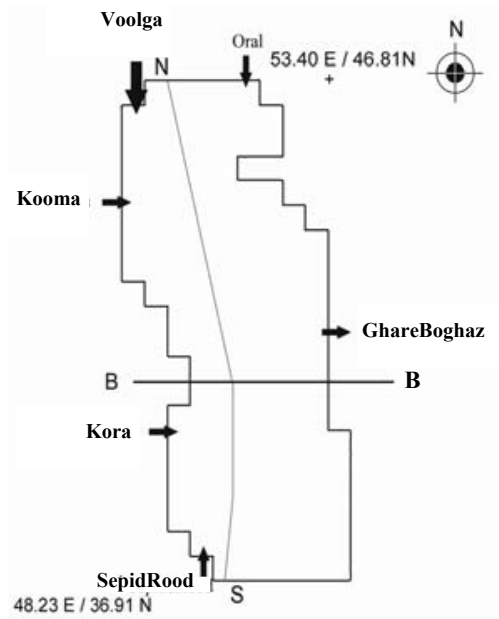

Figure 3: $\quad$ Boundary and reticulating according to numerical method. 


\section{Reticulation and field conditions}

Horizontal reticulation of the surface covers a $10 \times 10 \mathrm{Km}$ area. The vertical distance of ten horizontal layers has been assumed as variable. The equation covers all the knots in the grid, 19489 spots knots, being in the different layers. The distribution of the knotty spots knots is as follows:

First layers 3512, second layers 3006, third layers 2519, fourth layers 2377 , fifth layers 2178, sixth layers 1706, seventh layers 1424, eighth layers 1214 , ninth layers 991, and tenth layers 564. The process is chosen from 0 to 6 o'clock, 6 to 12 in time steps $\Delta \mathrm{t}=3600$ second. The distribution function is measured and the horizontal velocity, when repeated, can be measured by equation (8). As the explicit method has been used, all the location properties are approximated through the central difference method. To make the system convergent, the discussion of stability appears. The stability case is evaluated as $\Delta t<\frac{\Delta x}{C \sqrt{2}}$ through [3]. $\Delta t$ equals the time relation according to second, $\Delta x$, represent the location distance of the spots knots on the grid, and $\mathrm{C}$ equals the speed of flow according to meter/second. To determine the primary temperature field, the $15^{\text {th }}$ September 1995 data and reports of northern, southern and central areas of the Caspian Sea have been considered. The new temperature using Adams-Beshfort's method through the equation number 4 has been measured [5]. At the bottom of flux, the temperature is zero. The stress is defined by the Drag coefficient method. The viscosity and Eddy diffusivity as well as thermal diffusivity have been evaluated through the formula of monk-Anderson $[1,5]$.

$$
\begin{gathered}
A=100\left|\tau / \rho_{\circ}\right| \frac{1}{\left(1+10 R_{i}\right)^{3 / 2}} \\
K=100\left|\tau / \rho_{\circ}\right|\left[\frac{1}{\left(1+\frac{10}{3} R_{i}\right)}\right]^{\frac{1}{2}} \\
R_{i}=\frac{g}{\rho}\left(\frac{\partial \rho}{\partial z}\right) /\left(\frac{d V}{d z}\right)^{2} \\
A_{x}=A_{y}=0.25 f \min ^{2}(\Delta x, \Delta y)
\end{gathered}
$$

The wind stress at the surface is used by the formula.

$$
\tau_{w}=\rho_{a} c_{d}|\mathrm{~W}| \mathrm{W}
$$

in which $\mathrm{C}_{\mathrm{d}}$ represents Drag coefficient that equals $\rho_{a}$ and $c_{d}=(0.8+0.065)\left|W_{10}\right| \times 10^{-3}$ the air density equals $1.223 \mathrm{~kg}^{-3}$. The bottom stress has used the formula:

$$
\tau_{b}=0.002 \rho_{\mathrm{o}}\left|V_{b}\right| V_{b}
$$

and the stress in the middle layers can be used as:

$$
\tau_{m x}=A_{z} \frac{\partial U}{\partial z}+A_{h} \frac{\partial W}{\partial x}
$$


In the above equation the first term results from the vertical changes of horizontal velocity causing the transportation the pure stress in the incomparable fluid. The second term results from the horizontal changes on the X-axis.

\subsection{The results of the Caspian Sea modeling}

Figures 4 and 5 demonstrate the pattern of wind field distribution of the surface of the Caspian Sea at 6 and 12 o'clock on September 15. The results obtained from the numerical analysis show that the Caspian Sea circulation is influenced by the variability of density, the wind, the bottom friction, rivers emptying, temperature difference, and the boundary of closed field.

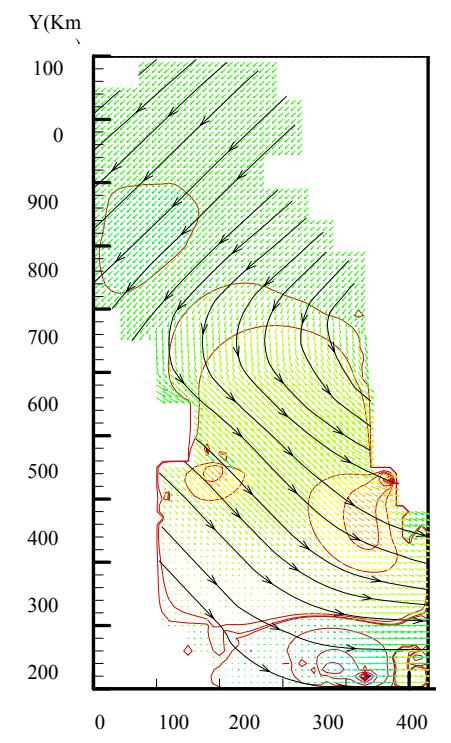

Figure 4: The pattern of wind field at 6 o'clock on September 15 has been constantly considered.

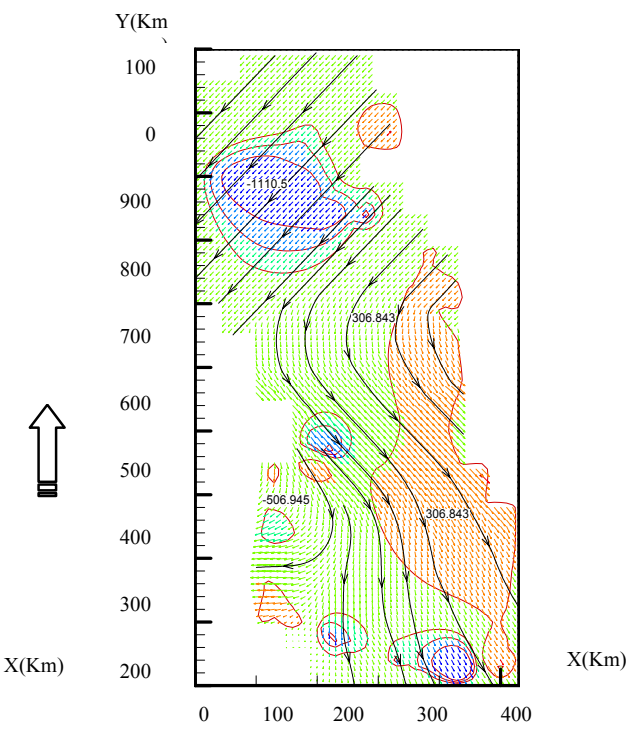

Figure 5: The pattern of wind field at 12 o'clock on September 15 has been constantly considered.

Figure 6 shows the flow distribution and its velocity in the first layer at 6 o'clock. The presence of the circular flows is visible in the northern and middle layers. The northern-eastern uprising in the middle area causes the water circulation to the left. Thus, combining with the waters moving from western coast of north to south, they form a complete circulation in the northern and middle areas. The flow velocity is variable throughout the area, ranging from almost $0.3 \mathrm{~mm}$ to $1.8 \mathrm{~cm} / \mathrm{s}$. Moreover, the current velocity, in comparison with northern and central areas, is less.

In the northern part of the Caspian Sea, as the result of shallowness, current velocity is radically reduced or becomes zero. A divergence of flow, when the 
mass water moves from the northern area to the central part, is obvious. At the western part of northern area, the water moves toward south and the wind circulation is from left to right (Figure 7).

Figures 6 and 7 show the profile of the changeability of the flow function and velocity, based on depth at the widthwise axis at 6 o'clock (figure 6). Around the middle part of the Caspian Sea two opposite cycles are visible throughout the widthwise axis having the positive and negative flow functions. The right part shows the eastern area of the Caspian Sea in the middle section. The wind field pattern, between 0 to 6 o'clock, is almost vertical on the shore. The flow function has become positive, then when the water is deep it is reduced.

This proves both the downwilling and convergence at this area. The reduction of velocity occurs at the deep location in which the water motion is downward resulting in the water downwilling.

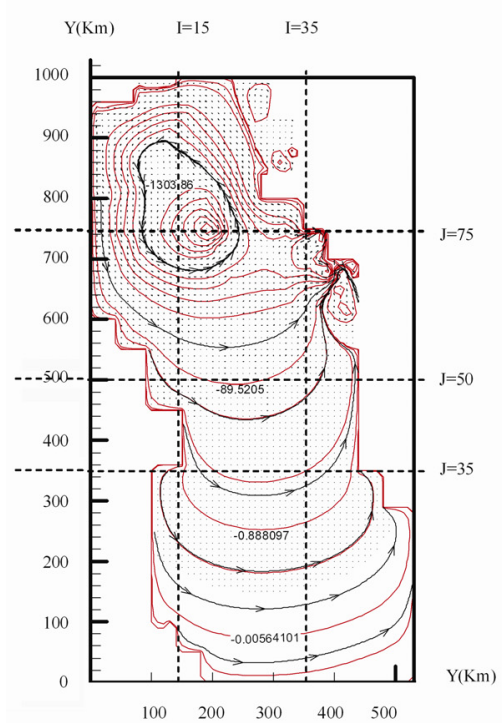

Figure 6: The distribution of stream function and velocity in the fourth layer at 6 o'clock gained from the numerical model.

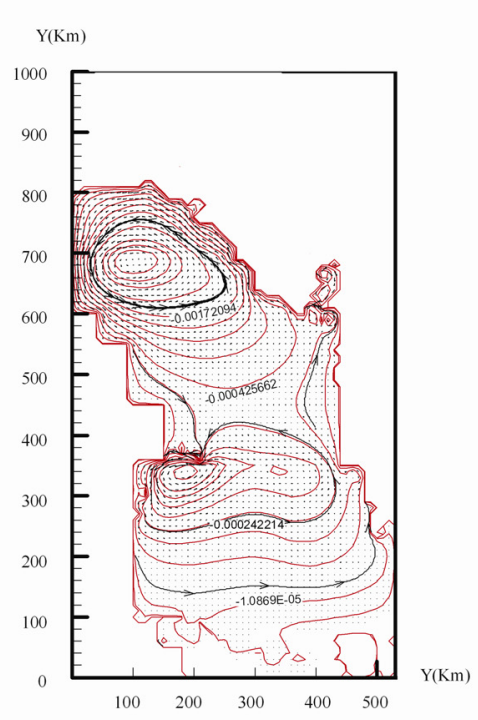

Figure 7: The distribution of stream function and velocity at the surface of the Caspian Sea at 6 o'clock gained from the numerical model.

In the fourth layer, as the result of shallowness of the northern part of the Caspian Sea, (in comparison with the southern and middle area), the velocity mentioned is considerably low, which is itself caused by the friction of the seabed. In the east of southern area, because the wind direction is often northwest, a flow is caused at the surface which after confronting the eastern and 
southern boundaries and meeting the western flow makes a complete circular flow from left to right which is even visible in the fourth layer. The current velocity at 12 o'clock has been measured from about $1.6 \mathrm{~cm} / \mathrm{s}$ to $15 \mathrm{~cm} / \mathrm{s}$.

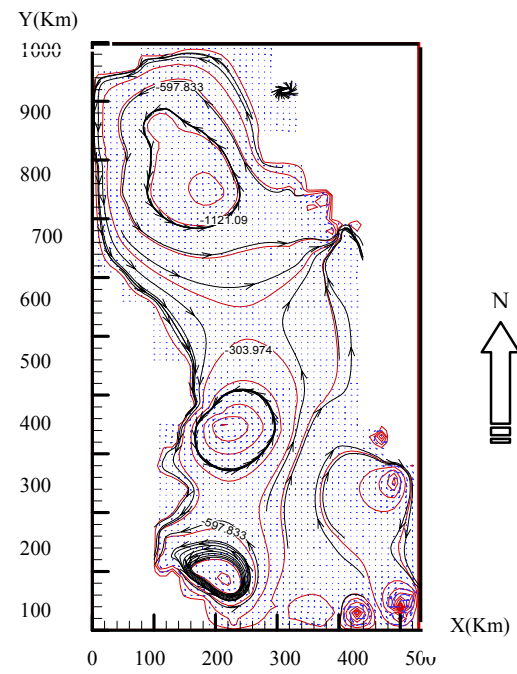

Figure 8: The stream function and velocity distribution at the surface the Caspian Sea in the four layers at 12 o'clock gained from the numerical analysis.

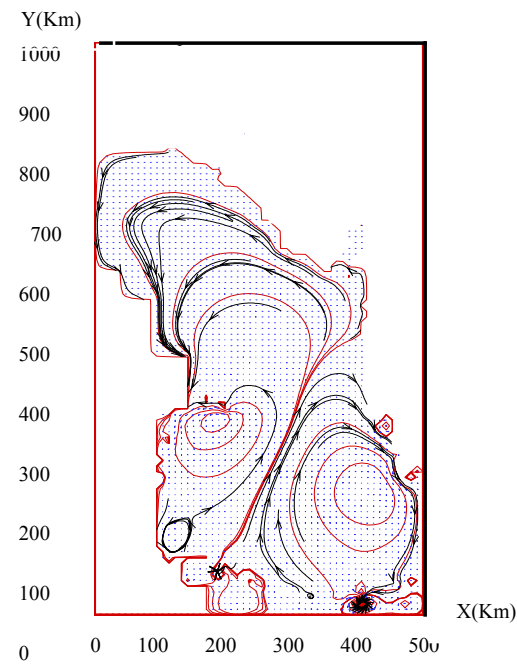

Figure 9: The stream function and velocity distribution at the surface the Caspian Sea at 12 o'clock gained from the numerical analysis.

\section{The result of the numerical analysis of the temperature and its comparison with the observation related to the sea research ship}

The results derived from the numerical analysis in the 0-50, 50-200, and 200-800 are as follows:

\section{0-50 meter layer:}

1. Generally, the decrease of vertical temperature in the mixed layer in the northern, middle, and southern parts.

2. The thickness of the thermocline layer at the station number 4 shows the highest point. It has decreased in the middle and northern parts from south to north.

3. Generally speaking, as the latitude increases, the temperature of water surface is decreased. Between the stations 1 to 9 , the horizontal heat gradient has 0.117 centigrade per kilometer. 
4. In southern part the vertical heat gradient in the layer being thermocline has followed two various gradients, while the changes in the middle and southern part of north have almost followed the stable gradient.

\section{0-200 meter layer:}

1. The water temperature has decreased from south to north.

2. The vertical heat gradient in southern part is more, in comparison with the middle and southern parts.

3. The sea bathymetry has affected the lines having the same temperature.

4. The vertical heat gradient is remarkable in the southern and middle parts in comparison with the north part 200-800 layers.

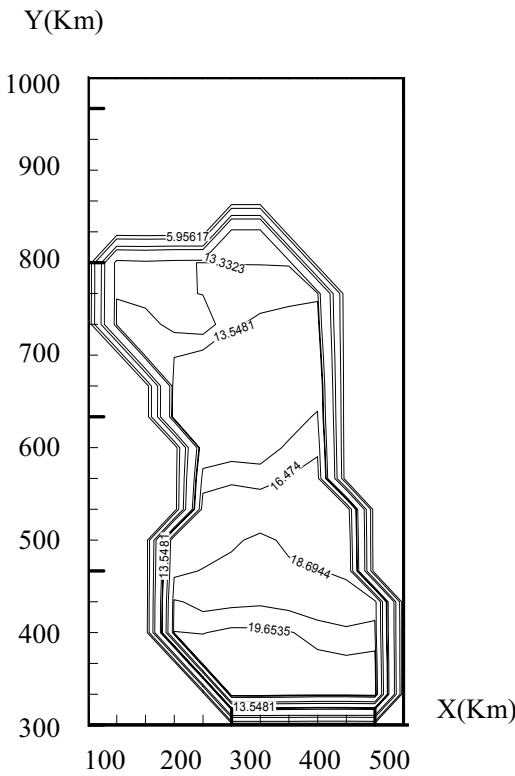

Figure 10: The distribution of thermal changes in the surface of the Caspian Sea, in the first layer, at the forecasted 6 o'clock.
$\mathrm{Y}(\mathrm{Km})$

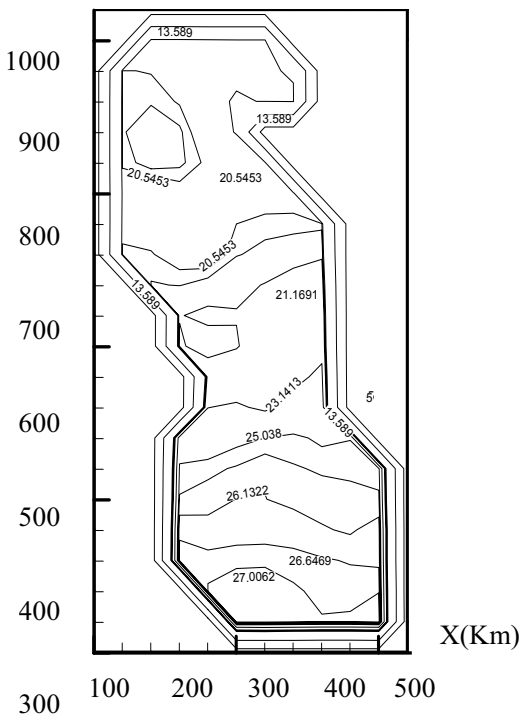

Figure 11: The distribution of thermal changes in the surface of the Caspian Sea, in the fourth layer, at the forecasted 6 o'clock.

1. In this layer the maximum of water topography in the southern, and middle parts have been 1, 6 respectively. The minimum of water temperature in these two parts has been 4.18 centigrade.

2. The vertical heat gradient in the middle part has been more than the one belonging to the southern part.

Briefly, it can be said that the model is able to show the Caspian Sea thermal structure desirably. These results show the considerable similarity between the reports of sea research ship and the present reports on the thermal structure of the Caspian Sea. 


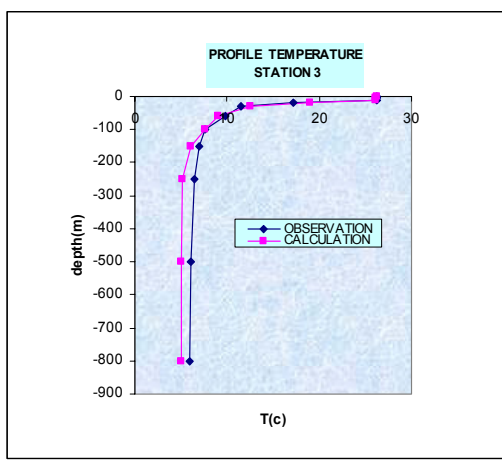

Figure 12: The comparison of the Figure 13: thermal profile derived from observation and numerical analysis at the station 7.

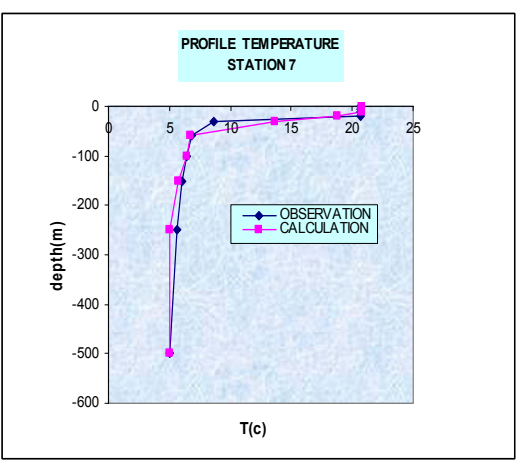

The comparison of the thermal profile derived from observation and numerical analysis at the station 2.

\section{Conclusion}

The present research can be considered as an example of the numerical analysis of the current which, in a constant condition, has been done by Bnnazadeh et al. [5]. I have something in common with Bennett and Birchfield's studies on Michigan and Ontario lakes [3].

The presence of clockwise anti cycles from right to left has been observed like the previous studies conducted in the northern, middle, and southern fields. This cycle conformity is in with the sea vertical cycle. The deep and surface element, in comparison with the previous studies, provide us with more information. This is because the model is time-related, and the wind pattern is changeable as well. Also, the smaller cycles derived from the numerical analysis of the model can be observed in the figures presented.

By considering the wind pattern between 0 and 6 o'clock, the presence of the flow cycles are clearly marked in the northern and middle fields. These cycles show the presence of mass water circulation from right to left. The current velocity in the surface of the first layer is between $3 \mathrm{~mm}$ to $1.8 \mathrm{~cm}$ at 6 o'clock. The maximum velocity belongs to the middle layer.

Current velocity in the first layers at 12 o'clock has been approximately $1.6 \mathrm{~cm} / \mathrm{s}$. it has been up to $15 \mathrm{~cm} / \mathrm{s}$ in the middle layer which maybe considered as the result of the stability of wind effects in this area. The survey of the lengthwise and widthwise cross section at 6 and 12 o'clock illustrates the dramatic decrease of velocity related to the depth. In the lower layers the vertical velocity at 12 o'clock is more than that of 6 o'clock. Moreover, in the lengthwise cross section a downwilling of water is observed in the northern parts of the Caspian Sea. Also, it appears as though there is a left to right circulation from north toward south in the middle area which is approximately 200 meters deep. 
The result derived from the numerical analysis of the temperature shows that its changes in eastern parts are more than its equal part in the western area. The lengthwise changes diagram of temperature distribution proves the presence of heat gradient from northern areas of the Caspian Sea to the ridge between southern in the upper ones. Water, in the lower layers of the southern field, is homogeneous. However, in the upper layers a heat gradient is observed at the middle of field towards the sides up to 200 meters depth.

In brief, if may be said that the present model has the capacity to show the thermal circulation and structure of the Caspian Sea in a large and medium scale. The result obtained from the model is in a good agreement with the information derived from research ship. By considering the experiments done on the numerical model and its results, it is possible to verify the correctness and accuracy of the stream function and velocity in the model presented.

\title{
List of symbols
}

\author{
$\boldsymbol{x}, \boldsymbol{y}, \boldsymbol{z}$ Cartesian coordinates, $\mathrm{m}(\mathrm{z}$, positive, downward) \\ $\boldsymbol{u}, \boldsymbol{v}, \boldsymbol{w}$ Velocity components $\mathrm{m}, \mathrm{s}(\mathrm{w}$, positive, downward) \\ $\boldsymbol{h}(\boldsymbol{x}, \boldsymbol{y})$ Depth of the Caspian Sea, $\mathrm{m}$ \\ p Pressure, without the surface pressure \\ $\boldsymbol{p}_{\boldsymbol{s}} \quad$ Surface pressure \\ $T \quad$ Temperature, ${ }^{\circ} \mathrm{C}$ \\ $R_{i} \quad$ Richardson number, without dimension
}

\section{References}

[1] Beletsky, D. \& Schwab. D.J., Modeling thermal structure and circulation in Lake Michigan, Estuarine and coastal Modeling, proceeding of the conference American society of civil engineers. Held October, pp. 22-24, 1977.

[2] Bennett, J.R., Three-dimensional model of Lake Ontario, summer circulation. J., Comparison with observations. J. Phys. Oceanogr., 7: pp. 591-601, 1977.

[3] Birchfield, G.F., Theoretical aspects of wind-driven currents in a sea or lake of variable depth with no horizontal mixing, J., Phys; Oceanogr. 2, pp. 355-366, 1972.

[4] Boyce, F.M., Donelan, M.A., Hamblin, P.F., Murthy, C.R. \& Simons, T. J., Thermal structure and circulation in the Great Lake, Atoms-ocean, 27(4), p. 607-642, 1989.

[5] Bnnazadeh, M., Baydokhti, A.A. \& Fadawi Hossaini, H., The primary survey of the three-dimensional model of the Caspian Sea, Tehran, Esteghlal magazine, 21, No. 1, 2001.

[6] Casandy, G.T., Lateral momentum finx in boundary currents, Phys. Oceanogr. 5, pp. 705-717, 1975. 
[7] Chubarenko, B.V., Wang, Y. \& Hutter. X., Wind-driven Current Simulation around the island Mainau (Lake Constance), Ecological Modeling, No 138, pp, 55-73, (2001).

[8] Kelley, J.G., HobGood, J.S. \& Schwab. D.J., Generation of threeDimensional Lake Model Forecasts for Lake Erie, pp. 659-687, 1998.

[9] Schwab, D.J. \& Bed ford, K.W., Initial implementation of the great Lakes forecasting system Areal time system for predicting lake circulation and thermal structure, water poll. Res. J., Canada. 29(213), pp. 203-220, 1994.

[10] Simons, T.J., Verification of Numerical Model of Lake Ontario: Circulation in spring and Early Summer. J., Phys. Oceanogr. 4, pp. 507-523, 1976.

[11] Wang, Y. \& Hutter, X., A Semi Inplicit-Spectral Primitive Equation Model for Lake Circulation Dynamics and its Stability Performance, J. Comput Phys. 139, pp. 209-241, 1998.

[12] The report of the international atomic energy agency at UNESCU, the survey of the Caspian Sea issues, Paris, May 9-12, 1995. 